\title{
Electric Linear Dichroism with a Simple Interpretation in Terms of Molecular Pendular States
}

\author{
A. Slenczka* \\ Institut für Physikalische und Theoretische Chemie, Universität Regensburg, D-93053 Regensburg, Germany
} (Received 1 August 1997)

\begin{abstract}
An anisotropic orientation of the figure axis of electrically dipolar $\mathrm{ICl}$ is forced by a strong homogeneous electric field. The molecule is transferred into a pendular state and shows optical anisotropy due to the body fixed molecular transition dipole moment. This holds in exactly the same way for magnetically dipolar molecules in a homogeneous magnetic field. A simple geometric model is applied which simulates all spectral features quantitatively without any fitting procedure for both the electric and the magnetic case. The sparse structure of polarization spectra of such a sample allows for easy reading of spectroscopic constants and study of electronic states of even large molecules. [S0031-9007(98)05653-1]

PACS numbers: 33.55.-b, 42.25.Ja, 42.79.Ci
\end{abstract}

For pendular states the free rotation of a dipolar molecule changes into a nonuniform rotation and finally into a small-angle libration if the molecule is put into a strong homogeneous electric or magnetic field [1]. The dipole moment and thus the molecular axis is aligned or even oriented in the laboratory frame. This goes hand in hand with an anisotropic orientation of the body fixed dipole transition moment. This aspect of pendular states implicated a polarization spectroscopic experiment for magnetic pendular molecules [2]. Signal generation is solely due to the anisotropy of molecular orientation which causes optical anisotropy in an absorbing or dispersive sample. The well defined polarization of incident light is rotated by the anisotropic absorption of the sample. The high sensitivity of this technique is based on the suppression of noise and background terms in the signal by orders of magnitude [3]. The contrast to a Schawlow-Hänsch type polarization experiment [3] optical anisotropy is created by a dc field instead of a pump laser. A circularly polarized saturating pump laser changes the population among degenerate $M_{J}$ sublevels [4] which causes birefringence or dichroism. The dc field, however, forces the molecular dipole of all molecules to orient itself parallel to the field direction. Only the hindered free rotation of the molecular dipole in the external field is considered in this work. Field induced dipole moments do not appear at our experimental conditions but are considered in the discussion. In a magnetic field of low field strength this type of spectroscopy presented in Ref. [2] coincides with magnetic rotation spectroscopy (MRS) [5].

The generated signal is due to the rotation of the plane of polarization of the transmitted laser light. We do not include the phase of the laser wave in our model calculations, and consider the case of a magnetic or electric dc field transversal with respect to the laser beam axis (Voigt- or Kerr-effect geometry). Our interpretation is based on differential absorption of light polarized parallel (for $\Delta M=0$ ) or perpendicular (or $\Delta M= \pm 1$ ) with respect to the dc field whose direction coincides with the average direction of the figure axis of the molecular pendular states. Since the linearly polarized input laser field $E_{0}$ (as shown in the upper part of Fig. 1) is a coherent superposition of two plane waves $E_{\perp}$ and $E_{\|}$polarized parallel and perpendicular to the homogeneous external dc field, an anisotropic absorption results in different amplitudes of the two field components and thus in a rotation of the plane of polarization of their coherent superposition. The transmitted amplitude has a nonzero projection $E_{T}$ onto the second polarizer axis whose axis is perpendicular with respect to the incoming plane of polarization. It is then detected by the photodiode. This geometric interpretation of linear dichroism is illustrated in the upper part of Fig. 1. The sensitivity of the technique critically depends on the extinction ratio of the polarizers.

A slight deviation from the exact $90^{\circ}$ rotation of the second polarizer with respect to the first one by a detuning angle $\zeta$ allows the direction of rotation of the polarization plane to be determined. Now even $E_{0}$ has a component $E_{T_{0}}$ parallel to the second polarizer creating a base line signal level. Rotation of the polarization opposite to the detuning angle $\zeta$, as caused by a transition dipole moment polarized parallel to the external field, causes a positive signal as shown in the lower part of Fig. 1. A transition dipole moment polarized perpendicular to the external field results in a polarization rotation along the detuning angle which appears as a negative signal. This geometric interpretation can easily be expressed by the following equation:

$$
E_{T}=\left[E_{\|}^{2}+E_{\perp}^{2}\right]^{1 / 2} \sin (\xi \pm \zeta)+E_{0} \sin ( \pm \zeta),
$$

where $\xi=45^{\circ}-\arctan \left(E_{\|} / E_{\perp}\right)$ is the angle of rotation of the plane of polarization of the transmitted wave with respect to the incoming one. The squared convolution with an appropriate line shape function simulates the spectra. Identical results are obtained when treating the absorbed part of the light. This is visualized by the vectors originating from the top of the incoming amplitude $E_{0}$ in both 

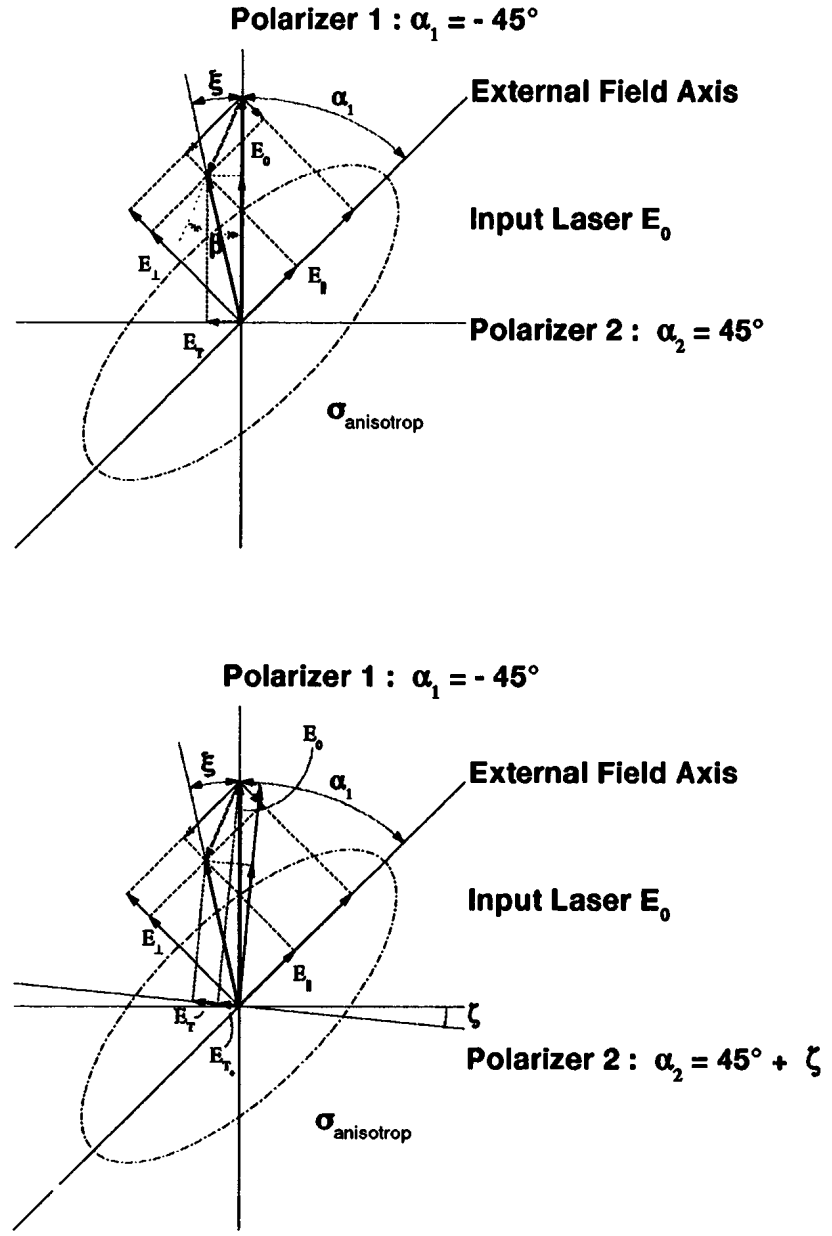

FIG. 1. Vector diagram for polarization rotation spectroscopy. Incoming electromagnetic wave polarization denoted by $E_{0}$; transmitted wave polarization by $E_{T}$; angle of rotation of the plane of polarization by $\xi$. Upper part of the diagram pertains to polarizers $\mathrm{P} 1$ and $\mathrm{P} 2$ crossed by exactly $90^{\circ}$. In the lower diagram the second polarizer $\mathrm{P} 2$ detuned by $+\zeta$ and the transmitted wave $E_{T_{0}}$ is the base line. Both diagrams show an anisotropic absorption coefficient $\sigma_{\text {anisotrop }}$ (dashdotted ellipse). See text.

the lower and the upper parts of Fig. 1. Taylor series expansion and truncation after the linear term reduces the expression for the absorbed part to the respective line strength factor $S(n ; \Delta M= \pm 1,0)[2]$. The final equation used for the simulation of the experimental spectra is

$$
\begin{aligned}
E_{T}= & {\left[S(\Delta M= \pm 1)^{2}+S(\Delta M=0)^{2}\right]^{1 / 2} } \\
& \times \sin (\delta \pm \zeta)+E_{0} \sin ( \pm \zeta),
\end{aligned}
$$

with $\delta=45^{\circ}-\arctan [S(\Delta M=0) / S(\Delta M= \pm 1)]$. The last term in Eqs. (1) and (2) is the base line transmission $E_{T_{0}}$ which will be discussed below. Calculation of the respective line strength factors for pendular states using quantum mechanical standard methods [1] and squared convolution of Eq. (2) with an appropriate line shape function is a simple procedure, applicable at any field strength and for any detuning angle. Guided by the simple picture of pendular molecules aligned parallel to the external dc field Eq. (2) is nothing but the resultant anisotropy of the absorption without phase information. For a dipolar molecule the interaction parameter $\omega=\mu F / B$, which measures the maximum potential energy of the dipole $\mu$ in the external field $F$, is readily calculated from the measured dc field strengths and the known molecular constants of ICl [6,7]. The calculations do not involve any fitting parameters. Since this worked so well for magnetic pendular states [2], the electric analog deserves attention. Curiously enough, this effect known as electric dichroism has been applied with rather complicated pulsed or sinusoidal modulated high voltage electric fields or modulated input polarization $E_{0}(t)$ using phase sensitive detection schemes [8]. The signals generated by modulation techniques are derivatives of the electric dichroism of the sample with respect to the modulated parameter. Nonmodulated polarization experiment [2] has been reported for gas phase samples in the field of electro-optical spectroscopy only rarely as a less sensitive technique which does not distinguish between the various $\Delta J$ branches [8]. Our concept brings out the identity of magnetic rotation and electrooptical spectroscopy. Furthermore, the theoretical concept of pendular states provides a very simple and vivid simulation procedure for polarization spectroscopy on dipolar molecules in a homogeneous field.

The experimental setup is described elsewhere [2]. Only the magnet is replaced by a stainless steel condenser inside a sample cell in order to study electric dipolar molecules. No phase sensitive detection technique or any modulation of experimental parameters is necessary. The upper trace in Fig. 2 shows the experimental spectrum with the pair of polarizers crossed by exactly $90^{\circ}$. The $\mathrm{cw}$ dye laser has a power of $300 \mathrm{~mW}$ and path length through the sample of $20 \mathrm{~mm}$. The field strength $E$ is $3.5 \mathrm{kV} / \mathrm{cm}$. At this field

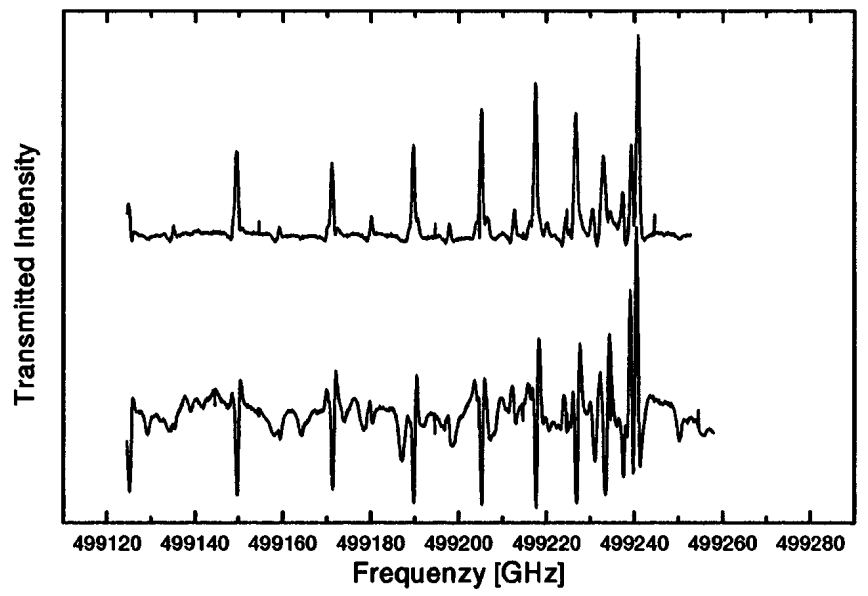

FIG. 2. Polarization spectra of the ICl $\left[A^{3} \Pi_{1}(19)-X^{1} \Sigma(0)\right]$ electric pendulum bandhead at electric field of $3.5 \mathrm{kV} / \mathrm{cm}$. Upper spectrum: with exactly $90^{\circ}$ crossed polarizers. Lower spectrum: second polarizer detuned by $+1^{\circ}$ from exactly $90^{\circ}$ crossed position. 
strength the spectral features appear at the same frequency position as the calculated line positions of the field-free ICl $A^{3} \Pi_{1}(19)-X^{1} \Sigma(0)$ rotational band transitions except for the lowest rotational states in the bandhead. The signal to noise ratio is orders of magnitude larger than for ordinary absorption spectra of $\mathrm{ICl}$ (see Ref. [2]) as is reported for polarization spectroscopy [3,9]. The $Q$ branch dominates in intensity while the $P$ and $R$ branches nearly disappear in the spectrum except for the lowest rotational states within the bandhead. The intensity distribution within the $Q$ branch shows a maximum for $J^{\prime \prime}=4$ expected at $3 \mathrm{~K}$ in a thermal sample. However, the maximum population density of the ICl room temperature sample is at $J^{\prime \prime}=30$. At lower field strength the maximum line intensity shifts to even lower $J$ states. Because of this low $J$ selectivity and, in addition, because of the $\Delta J$ selectivity band origins and rotational constants are easily read from the spectra [2]. At higher field strengths line intensities may behave irregularly due to nonmonotonous change of the eigenenergy of pendular states $[1,10,11]$. In this Letter we present spectra for only one value of field strength. However, the field strength dependence has been investigated in detail for the magnetic analog of this experiment [2]. It certainly behaves in exactly the same way for the electric case, because first the simulation procedure works equally well for both magnetic and electric dipolar molecules for our experiment, and second this identity was also found for fluorescence excitation spectra of pendular states [6] where both electric and magnetic field strengths have been varied.

The experimental observation for slightly detuned polarizers is shown in the lower part of Fig. 2. It covers the same bandhead of ICl. At the same electric field strength the detuning angle $\zeta$ of the second polarizer is $+1^{\circ}$. For nonoverlapping transitions the line shape changes from a single positive peak into either a negative line center and positive shoulders or vice versa. The first line shape represents the $Q$ branch, the latter the $P$ and $R$ branches. At this field strength only the phase of the lowest transition of the $R$ branch can be recognized as opposite to the $Q$ branch. Other $P$ and $R$ lines are hidden due to overlapping transitions or due to the $\Delta J=0$ selection. The line shape is an unequivocal indication for the $|\Delta J|$ of the respective transition. The background signal level increases by orders of magnitude compared to the upper spectrum in Fig. 2 and shows, in addition, simple isotropic absorption structures from hot $\mathrm{ICl}$ or $\mathrm{I}_{2}$.

Further investigations were done on the $B^{3} \Pi_{0}(3)-$ $X^{1} \Sigma(1)$ band head of $\mathrm{ICl}$ for $90^{\circ}$ crossed polarizers. Both electronic states of this $\mathrm{ICl}(B-X)$ transition are plane rotor states $(K=0)$ exhibiting no first order field effect. An electric field strength of about $7 \mathrm{kV} / \mathrm{cm}$ which produces interaction parameters $\omega$ of about 1.3 and 1.4 for $X$ and $B$ states, respectively, induces negligible energy splitting of the $M_{J}$ levels on the order of $10^{-3} \mathrm{~cm}^{-1}$. Since this splitting correlates with the anisotropy [1], the polarization spectroscopic signal is much lower than for the $A^{3} \Pi_{1}$ state. This is also found for fluorescence excitation spectra of the $B-X$ transition taken in a supersonic beam where significant effects appear only at field strengths larger than $10 \mathrm{kV} / \mathrm{cm}$ [12]. Furthermore, the absorbing state is the first vibrationally excited state of $\mathrm{ICl}$, an order of magnitude lower in population density than the vibrational ground state. Even so we were able to detect very few lines of the bandhead with a signal to noise ratio of about 5 . The polarization spectra of ICl pendular states can easily compete with Stark modulation (SM) spectra [13]. The use of dc electric field is much simpler experimentally as well as for simulations, and no phase sensitive detection is required.

Simulations of the experimental results using Eq. (2) are shown in Fig. 3. The calculations do not involve any fitting parameters. Spectral structures, intensities, and phases are quantitatively reproduced for both the $90^{\circ}$ crossed as well as the $91^{\circ}$ crossed polarizers. Minor deviations of the experiment are caused by inhomogeneities in the field strength over the path of the laser through the condenser while calculations are performed for a fixed value $\omega$. Taking $\omega$ as a fitting parameter allows one to measure either field strengths with local resolution or even to determine molecular dipole moments. Comparison of simulation and experiment for $90^{\circ}$ crossed polarizers shows small negative signals in the experimental spectrum which do not appear in the simulation. This is due to a deviation of the crossing angle from exact $90^{\circ}$. The lower trace of the simulation in Fig. 3 does not show the residual isotropic absorption of the sample. This can be included by replacing the constant factor $E_{0}$ in Eq. (2) by the simple absorption of the sample. Nevertheless, since the dichroitic part dominates the spectrum even a constant $E_{0}$ simulates the experiment in this case very well.

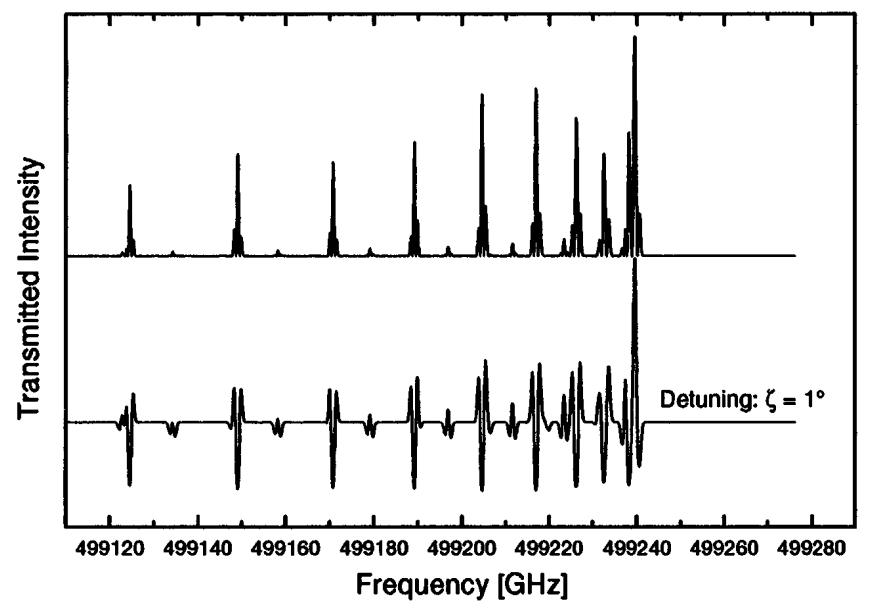

FIG. 3. Simulated polarization spectra for ICl pendular states. Interaction parameter $\omega^{\prime}=1.4$ and $\omega^{\prime \prime}=1.3$. Upper part: detuning angle $\zeta=0$. Lower part: detuning angle $\zeta=1^{\circ}$. Both spectra simulate the experimental results presented in Fig. 2. 
The method of producing optical anisotropy of transition moments in a molecular gas phase sample with dc homogeneous fields can easily be extended to any molecule with a body fixed electric dipole moment. As the results show, all the advantages known from magnetic rotation spectroscopy [5] like low $J$ and $\Delta J$ selectivity appear in polarization spectra of electric pendula. In contrast to the magnetic case with a free rotor ground state $[2,6]$ the electric dipolar $X^{1} \Sigma$ state of ICl shows pendular motion which at this field strength causes a signal enhancement only for the very first lines in the bandhead. Otherwise the spectral structures are identical for the magnetic case at the same interaction parameter $\omega^{\prime}$. However, the electric analog to the Faraday effect does not exist. The optical anisotropy and circular dichroism in the Faraday-effect geometry is caused by right and left hand circularly polarized dipole transition moments of the $\Delta M=+1$ and $\Delta M=-1$ transitions. In the electric case it cancels exactly due to the $|M|$ degeneracy. This originates from the different symmetry character of electric and magnetic dipole operators which exhibits different degeneracy for electric and magnetic pendular states $[6,11]$. The prototype $\mathrm{ICl}$ molecule has the advantage to serve as an electric, as well as magnetic, dipolar pendulum. This is the ideal condition for comparing related physical effects with a minimum change of parameters. The results are striking. All polarization spectra of all kinds of molecular pendula can be simulated and interpreted in terms of the anisotropy of the figure axis correlating with anisotropic dipole transition moment. Famous electro-optical gas phase experiments as of Buckingham [8] are very similar to our experiment. However, Buckingham, who presents very few similar spectra [8] like ours with lower spectral resolution, preferred a variety of modulation techniques with different optical setups. He even states that the technique of our experiment does not distinguish different $\Delta J$ transitions which is in contrast to our findings. Electric field modulation (Stark modulation spectroscopy) or modulation of the polarization in connection with phase sensitive detection show that the obtained signals are derivatives of our signal with respect to the modulated parameter. It is well known that this derivative depends nonlinearly and nonmonotonously on the field strength $[1,10,11]$. Therefore, the simulation and interpretation of our experiment is much easier and straightforward. However, Buckingham's much more sophisticated experiments allow one to distinguish between birefringence and dichroism. Finally, it is common knowledge that modulation techniques increase sensitivity by orders of magnitude which holds also for our experiment in case of modulating the laser intensity.

As has been shown theoretically $[11,14,15]$ and experimentally $[16,17]$ even nonpolar molecules are accessible for this technique. Induced dipole moments created by the external field and an anisotropic polarizability induce optical anisotropy in exactly the same way. This extension of the experimental technique generally allows detection of spectra from gas phase samples at high temperature that reveal better selectivity and sparser spectral structures than those taken from samples at very low temperatures. Moreover, in combination with molecular beams the technique should provide a powerful tool to study electronically excited states of even polyatomic symmetric or asymmetric top molecules independent of fluorescence quantum yield. Experimental investigations on this are in progress in our laboratory.

We are indebted to Dr. habil. Jan Michael Rost for providing the computer code for calculating eigenenergies and line strength factors for pendular states.

*Electronic address: alkwin.slenczka@chemie.uni-regensburg.de

[1] B. Friedrich and D. R. Herschbach, Nature (London) 353, 412 (1991); B. Friedrich, D. R. Herschbach, J.-M. Rost, H.-G. Rubahn, M. Renger, and M. Verbeek, J. Chem. Soc. Faraday Trans. 89, 1539 (1993).

[2] A. Slenczka, J. Phys. Chem. 101, 7657 (1997).

[3] C. Wieman and T. W. Hänsch, Phys. Rev. Lett. 36, 1170 (1976); J. J. Song, J. H. Lee, and M. D. Levenson, Phys. Rev. A 17, 1439 (1978).

[4] W. Demtroeder, Laser Spectroscopy (Springer, Berlin, Heidelberg, New York, 1982).

[5] I. Hirano, Phys. Rev. A 50, 4650 (1994).

[6] A. Slenczka, B. Friedrich, and D. R. Herschbach, Phys. Rev. Lett. 72, 1806 (1994); B. Friedrich, A. Slenczka, and D. R. Herschbach, Can. J. Phys. 72, 897 (1994).

[7] G. Herzberg, Spectra of Diatomic Molecules (Van Nostrand, New York, 1950).

[8] A. D. Buckingham and D. A. Ramsay, J. Chem. Phys. 42, 3721 (1965); J. M. Brown, A. D. Buckingham, and D. A. Ramsay, Can. J. Phys. 49, 914 (1970); D. C. Clary and B.J. Orr, Optical Electric and Magnetic Properties of Molecules, A Review of the Work of A.D. Buckingham (Elsevier, Amsterdam, 1997).

[9] B. Dick, Chem. Phys. Lett. 143, 186 (1988); Chem. Phys. 136, 413 (1989); 136, 429 (1989).

[10] E. U. Condon, Phys. Rev. 31, 891 (1928).

[11] A. D. Buckingham, MTP Int. Rev. Sci. Phys. Chem. Ser. One 2, 241 (1972).

[12] A. Slenczka, B. Friedrich, and D. R. Herschbach, Chem. Phys. Lett. 224, 238 (1994).

[13] A. Watanabe, T. Takei, and Y. Amako, Jpn. J. Appl. Phys. 31, 901 (1992).

[14] B. Friedrich and D. R. Herschbach, Phys. Rev. Lett. 74, 4623 (1995); B. Friedrich and D. R. Herschbach, J. Phys. Chem. 99, 15686 (1995).

[15] E. U. Condon, Phys. Rev. 41, 759 (1932).

[16] M.F. Crawford and R.E. MacDonald, Can. J. Phys. 36, 1022 (1958); T.W. Terhune and A.D. Dows, J. Mol. Spectrosc. 3, 138 (1959).

[17] W. Kim and P. M. Felker, J. Chem. Phys. 104, 1147 (1996). 